\title{
Pollen sensitisation profiles of allergic patients in a Central European region
}

\author{
Petr Panzner ${ }^{1 *}$, Tomas Vlas², Martina Vachova ${ }^{2}$, Petra Vitovcova ${ }^{2}$ \\ From 5th International Symposium on Molecular Allergology (ISMA 2013) \\ Vienna, Austria. 6-7 December 2013
}

\section{Objective}

The aim of our study was to assess the pollen sensitization patterns by means of molecular diagnosis approach in the region of Pilsen, Czech Republic.

\section{Methods}

The microarray system ImmunoCAP ISAC has been used for specific IgE detection to 113 different allergenic molecules. Sera from 789 patients sensitized to at least one pollen-derived molecule were subject of analysis. These patients suffered from at least one of the following diagnoses: chronic rhinitis (62\%), bronchial asthma (33\%), atopic dermatitis (28\%), urticaria or angioedema (28\%) and/or anaphylaxis (11\%). Patient age ranged from 2 to 68 years, with a mean age of 32,4 years. The sex ratio was $37 \%$ men to $63 \%$ women.

\section{Results}

The most frequent sensitization rate was observed to grass-derived species-specific molecules (82,2\% overall), the most frequent being Phl p 1 (66,3\%), markedly overwhelming sensitization rates to any non-pollen-derived molecule. The second one were pollen-derived PR-10 molecules $(53,2 \%$ overall), of which the large majority included Bet v 1 (52,3\%). Sensitization to these two types of pollen components (and their co-sensitizations with other components) forms the vast majority of pollen sensitizations. The patterns of co-sensitization is presented by means of Venn diagram approach. Sensitization to Cupressaceae-derived molecules was observed in $16,0 \%$ of subjects, to Oleaceae derived-molecules in 12,5\% (Ole e 1 and Ole e 9 in $9,0 \%$ and 3,8\% resp.) and to the plane treederived molecules Pla a 2 and Pla a 3 in 15,4\% and 4,1\% resp; these relatively high rates were surprising as the respective pollens have not been considered as important in the region. The sensitization rates for further molecules were: Art v 1 - 13,4\%, Pla $11-11,4 \%$, Che a $1-9,9 \%$, Par j $2-0,9 \%$, Sal k $1-0,6 \%$ and Amb a $1-0,3 \%$. The sensitization rates to cross-reacting molecules were generally not as high as reported from other regions (profilins $16,2 \%$, polcalcins $5,5 \%$, LTPs $6,7 \%$ ). Only $1,8 \%$ patients reacted to pollen-derived panallergen and not simultaneously to a pollen species-specific component.

\section{Conclusion}

Molecular diagnosis of allergy gives a more precise and comprehensive insight into pollen sensitization patterns than extract-based testing, allowing for better understanding of the sensitization process and regional differences. The data presented may help to improve diagnostic and treatment specific procedures in the respective region.

\section{Authors' details \\ ${ }^{1}$ Dept. of Immunology and Allergology, Medical Faculty in Pilsen, Charles University Prague, Alej Svobody 80, Pilsen, 30460, Czech Republic. ${ }^{2}$ Charles University Prague, Medical Faculty in Pilsen, Dept. of Immunology and Allergology, Pilsen, Czech Republic.}

Published: 17 March 2014

doi:10.1186/2045-7022-4-S2-P38

Cite this article as: Panzner et al:: Pollen sensitisation profiles of allergic patients in a Central European region. Clinical and Translational Allergy 2014 4(Suppl 2):P38. 